LUBLIN STUDIES IN MODERN LANGUAGES AND

LITERATURE 39 (2), 2015, HTTP://WWW.LSMLL.UMCS.LUBLIN.PL

\title{
Reinhold Utri
}

University of Warsaw, Poland

U1. Szturmowa 4, 02-678 Warsaw

\section{Das Medium Film als Element mit hohem interkulturellen Potenzial im DaF-Unterricht - am Beispiel der Filme Solino und I love Vienna}

\begin{abstract}
For some years now scientists have been dealing with multi- and intercultural phenomena. After presenting definitions of interculturalism and the integration policy in Germany for foreigners in the 70s, the author states that nowadays teachers start to understand the problem of migration and therefore multicultural and -lingual classrooms better. Many teachers try to take advantage of an additional culture/ language as a certain value. How to start dealing with those subjects and to motivate students to think these topics over is shown on the example of two films, a German one (Italian immigrants and their life for twenty years in Germany) and an Austrian film from the early 90s (an Iranian guy, former German teacher in his home country, comes to Vienna, taking his teenaged son and sister with him and tries hard to start a new life in Vienna. It is stated that a teacher can work with a film as a medium to reach his goal of discussing the current global topic foreign cultures.

Keywords: interculturality, multiculturality, film, immigrant, islam, Vienna, integration, Europe, culture, language
\end{abstract}

1. Multikulturalität 
Das Medium Film als Element mit hohem interkulturellem Potenzial 155

Die Ausdrücke Multi- bzw. Interkulturalität und interkulturelles Lernen - auf die nähere Definition dieser Begriffe wird untenstehend eingegangen - wurden nicht nur zu modischen Schlagwörtern in der Politik (siehe dazu J. Nowak, 2012), sondern gehören mittlerweile auch zum gängigen Wortschatz in wissenschaftlich-didaktischen Überlegungen (vgl. z.B. M. Mackiewicz 2005 oder R. Utri 2007a, 2010, A. Mucha, 2014). Das Bewusstsein, dass wir in einer multikulturellen Gesellschaft leben, existiert schon einige Jahrzehnte lang. So beschrieben die Engländer als erste ihre „culturally plural society" . Wie dieser Ausdruck andeutet, bedeutet er, dass in einem bestimmten Gebiet/Land/in einer gewissen Gesellschaft mehr als eine Gruppe lebt, die sich von anderen zumindest in ihrer Kultur unterscheidet ${ }^{2}$.

Der Ausdruck Multikulturalität (oder salopp bzw. abwertend Multikulti genannt) umfasst also die Tatsache, dass in einer Region/in einem Land eine hohe Diversivität an Kulturen existiert; anders ausgedrückt ist die Immigrantendichte, d.h. die Anzahl der Einwohner, die ausländische Wurzeln (egal ob sie aus einem anderen Land stammen oder ob sie Eltern/einen Elternteil haben, die/der z. B. immigriert ist und sie schon im Land geboren sind) aufweisen, so hoch, dass der Einfluss fremdländischer Mitbürger bemerkbar ist. Dies äußert sich nicht nur darin, dass fremde Sprachen auf der Straße gehört werden, sondern dass diese Immigranten sich sozial, sprachlich oder auch religiös-kofessionell organisieren (in Kulturvereinen etc.) oder dass auch für sie etwas von der Mehrheitsgesellschaft organisiert wird (z. B. Deutsch-Kurse für Ausländer).

In der Zuwanderungs- und Integrationsdebatte erklärte Angela Merkel im Jahre 2010, dass Multikulti gescheitert sei ${ }^{3}$. Dies kann jedoch gar nicht der Fall sein, da die unterschiedlichen Kulturen entweder in einem Land leben oder nicht. Einheimische und

\footnotetext{
${ }^{1}$ http://www.immi.se/intercultural/

${ }^{2}$ Vgl. J. M. Pomorski (2006: 71)

${ }^{3}$ http://www.spiegel.de/politik/deutschland/integration-merkel-erklaertmultikulti-fuer-gescheitert-a-723532.html (Zugriff: 12.10.2015
} 
Immigranten können relativ getrennt voneinander leben, es kann eine Art Ghetto entstehen, was zur Folge hat, dass viele Immigranten mit den Einheimischen kaum Kontakt haben und auch oft deren Sprache nicht/nicht gut lernen (türkische Viertel in Berlin, Polen in New York).

Multikulturalität erklärt ja nicht, wie viele Kontakte es zwischen einzelnen Gruppen (ethnische Gruppen, Eingeborene/Zugezogene, alte Einwohner/Immigranten) gibt, wie lange sie andauern bzw. wie intensiv sie sind; auch nicht, ob die Zusammenarbeit reibungslos funktioniert oder ob es zu häufigen oder ernsthaften Konflikten kommt. Hier hat die deutsche Kanzlerin die Begriffe Multikulturalität und Integration verwechselt.

\section{Interkulturalität}

Diese prozesshaften Aspekte der zwischen den Gruppen entstehenden Zusammenarbeit können in den Begriff Interkulturalität einverleibt werden. Alle interkulturellen Projekte/Tätigkeiten, die sich jemand aus einer Gruppe oder auch Drittpersonen (Kulturverantwortliche, Beamte usw.) ausdenken, haben - so wird es zumindest meist formuliert (und nehmen wir einmal an, dass dies alles ernst gemeint ist) - ein hehres Ziel: die guten Kontakte zwischen den Menschen mit unterschiedlichem kulturellen (und damit auch sprachlichen) Hintergrund zu vermehren, zu vertiefen, zu intensivieren.

Der Begriff Interkulturalität beschreibt also nichts Statisches, sondern Prozesse der Begegnung von Menschen mit unterschiedlichen kulturellen Hintergrund, wobei diese mehr oder weniger intensiv sein können. Dieses Aufeinandertreffen beginnt mit gegenseitigen Beobachtungen: wie verhalten sich die Türken/Serben/Araber/Schwarzafrikaner usw. in Deutschland? Wie gut/schlecht passen sie sich der Mehrheitsgesellschaft an (Kleidung, Benehmen, aber auch Sprache)? Sowohl die Immigranten als auch die Einheimischen erleben Fremdes, das ihnen anfänglich eigenartig vorkommt, das sie erst verstehen lernen. Vor allem auch die Immigranten können, falls sie eine gewisse Offenheit zeigen, dazu beitragen, dass die Einheimischen sich für die Kultur 
Das Medium Film als Element mit hohem interkulturellem Potenzial 157

(Hochkulturprodukte wie Gesang, Musik, Literatur; aber auch Alltagskultur wie Sitten und Bräuche) und nicht nur für einen kleinen Teil der gastronomischen Produkte (Kebab, Pizza, Sushi) interessieren. Die Migrantenliteratur liefert diesbezüglich einen wertvollen Beitrag (so schreibt Dilek Güngör über ihre türkische Familie, und dies humorvoll; Näheres zu Migrantenliteratur siehe R. Utri, 2010a, 2010c).

\section{Interkulturelles Lernen}

Bei all diesen gemischt-kulturellen Begegnungen und Aktivitäten ist interkulturelles Lernen möglich. Wenn jemand etwas über eine andere Kultur erfährt, so können wir das kulturelles Lernen nennen. Also wenn polnische Schüler über die deutsche Kultur lesen, lernen oder hören, so lernen sie kulturell. Wie der Begriff inter-kulturell schon aussagt, kommt es erst dann zum gegenseitigen kulturellen Lernen, wenn beide Seiten voneinander kulturell lernen; dies kann z. B. bei einem Austauschprojekt geschehen (polnische Schüler fahren nach Deutschland und deutsche nach Polen; dies kann auch nur virtuell über Mail etc. organisiert werden).

Idealerweise kommt es beim interkulturellen Lernen, so die gängige Meinung, zu einem Stereotypenabbau und zur Verminderung von alten, nicht mehr in die moderne Zeit passenden Mythen (über die andere Gruppe) und zur Demontage von Vorurteilen und Voreingenommenheiten. Die Hoffnung wird geäußert, dass es zwischen den jeweiligen Gruppen zu einer besseren Verständigung kommt. Die Voraussetzung dafür ist jedoch, dass die Menschen entsprechend wichtige (so genannte turning points of culture $^{4}$ ) Elemente, Sitten und Gebräuche sowie auch die kulturellen Werke der anderen Kultur kennen und schätzen lernen. Auch wenn dies geschieht, ist es oft eine traurige Tatsache, dass die Menschen sich für die Sprache (vielleicht der wichtigste Teil der fremden Kultur) nicht/kaum interessieren. Schon vor der Begegnung von fremden Kulturen (genauer gesagt von „Vertretern“ dieser) wäre es möglich

\footnotetext{
${ }^{4}$ Vgl. M. Brown, 1997.
} 
(und erwünscht), dass die Menschen von den fremden KulturElementen hören und die andere Sprache lernen, um schon von vornherein ein wenig von der anderen Kultur kennenzulernen und (sprachliches) Verständnis zu erreichen. Diese „Prophylaxe“ könnte idealerweise schon im Elternhaus, aber dann auch im Kindergarten/in der Schule beginnen. Allerdings ist es meist so, dass die Sprache des Landes den höchsten Stellenwert einnimmt und dass sich z. B. die Deutschen, um sich mit ihren Nachbarn, Taxifahrern, Ladenbesitzern, Kebab-Verkäufern zu unterhalten, nicht die Mühe geben, Italienisch, Türkisch, Serbokroatisch oder Arabisch zu lernen.

\section{Interkulturelles Potenzial}

Situationen der Begegnung von Immigranten und Einheimischen oder auch von unterschiedlichen Immigranten bergen also die Möglichkeit in sich, dass interkulturelles Lernen ermöglicht wird, dass das Interesse und das Verständnis für Elemente (Kunstwerke, Sitten, Mentalitäten) der anderen Kultur gefördert wird. Auch alle Materialien und Medien, die z. B. in der Sprachdidaktik (poln. Glottodydaktyka) verwendet werden können (z.B. Karikaturen, Näheres dazu siehe R. Utri 2013), haben dieses Potenzial, falls sie so genutzt und/oder aufbereitet werden, dass eben ein gegenseitiges kulturelles Kennenlernen und Lernen stattfinden kann (wobei nicht nur die didaktische Aufbereitung des Materials durch die Lehrperson, sondern auch noch andere Umstände dazu beitragen können und müssen).

5. Ausländerpädagogik

In den 1970er-Jahren sprach man in Deutschland von Ausländerpädagogik ${ }^{5}$; damals erkannten die Lehrkräfte, dass sie das sprachliche Defizit der Migrantenkinder abbauen müssten, um diese besser in die deutsche Gesellschaft zu integrieren. Die Lehrkräfte meinten es sicherlich gut, indem sie hofften, dass die Kinder dadurch

5 http://www.uni-koeln.de/ew-fak/paedagogik/interkulturelle/publikationen/ bern.html (letzter Zugriff: 12.06.2015). 
Das Medium Film als Element mit hohem interkulturellem Potenzial 159

später gute Chancen auf dem Arbeitsmarkt hätten, wenn sie eine ähnliche Sprachkompetenz wie ihre deutschen Altersgenossen aufweisen würden, wenn sie ähnliche Kleidung tragen oder sich ähnlich verhalten würden.

Dabei vergaßen sie allerdings, dass diese Kinder als Folge der „guten Integration“ den Kontakt mit ihrer Erstkultur immer mehr verlieren würden. Sie hätten also das Gefühl, dass sie durch die schlechteren Deutschkenntnisse schlechter wären und erst dann besser wären, wenn sie möglichst schnell ihre eigenen Wurzeln verleugnen würden, wenn sie ihre erste, für die Deutschen „exotische“ oder „eigenartige“ Sprache nicht benutzen und die Sitten ihrer Familie nicht mehr praktizieren würden.

\section{Leitkultur}

Ihre Länder/Heimat waren also etwas, worüber man gar nicht sprach oder ein Wort verlor. Diese fremde Kultur brachte hauptsächlich Probleme mit sich; so z. B. den Ramadan, der Fastenmonat (Näheres zum Fasten und zum Alkoholverbot vgl. R. Utri 2007:40, auch Lemmen/Miehl 2001) ${ }^{6}$. Während die Immigranten sich an ihrer Kultur/Religion festhalten, integrierten sich ihre Kinder ganz im Gegensatz zu ihren Eltern in die deutsche, „westliche“ (scheinbar homogene) Gesellschaft gut.

Viele Immigranten glaubten daran, dass ihre eigene Kultur (also auch ihre Muttersprache) minderwertiger als diejenige wäre, mit der sie jetzt kommunizieren mussten (vgl. http://www.nkjociechanow.edu.pl/...papa_hat_nichts_gegen_italiener_

\footnotetext{
${ }^{6}$ Darüber hinaus gibt es noch die Alkoholprohibition für Moslems und da der Alkoholkonsum im christlichen Kulturkreis weit verbreitet ist, ist die Abstinenz oft mit sozialer Ausgrenzung verbunden (v. a. unter Jugendlichen; siehe Jochen Schmidt über das Thema „Enthaltsamkeit macht einsam“, http://www.sueddeutsche.de/leben/ein-leben-ohne-alkohol-aussaufmodell1.1145023-2 (letzter Zugriff: 12.06.2015).
} 
3.pdf). ${ }^{7}$ Aufgrund dieser Einstellung verloren viele Migrantenkinder die große Chance auf Bilingualität und Bikulturalität, welche wir heute (im 21. Jahrhundert) so sehr schätzen. Das Phänomen doppelter Semilingualismus ${ }^{8}$ trat auf, und alle (Eltern, Lehrkräfte) waren relativ ratlos, was dagegen zu tun sei. Die meisten orientierten sich an der Leitkultur; dieser Begriff ist bis heute ein sehr umstrittener (vgl. http://www.welt.de/debatte...Warum-wir-ueber-die-Leitkultur-redenmuessen.html).

\section{Interkulturelle Pädagogik}

Erst später, wahrscheinlich unter dem Einfluss der „Dritten-WeltPädagogik" (dazu siehe z. B. B. Creemers, 1999, M. Wallenborn, 2007 sowie Quane/Singh, 2004) sowie auch der anti-rassistischen Erziehung kam es zur Interkulturellen Pädagogik. Der Fortschritt im Konzept dieser Richtung ist die Überlegung, dass es nicht mehr um die beste und schnellste Assimilation (also die Annahme der Sprache/Sitten des Gastgeberlandes ${ }^{9}$ ) geht. Der Wert der Kultur/Sprache der Immigranten werden nicht infrage gestellt ${ }^{10}$.

Lehrkräfte, die mit Menschen mit einem anderen kulturellen Hintergrund in Kontakt kommen, mit ihnen arbeiten, sie unterrichten, hätten eigentlich die Möglichkeit, den ganzen Reichtum, den diese „Fremden“ mitbringen (ihr kultureller/ sprachlicher Reichtum) zu berücksichtigen. Das hätte den großen Vorteil, dass erstens die Migrantenkinder ihr Selbstwertgefühl stärken würden, wenn ihre

\footnotetext{
${ }^{7}$ Dieses Thema, und das in Bezug auf Italiener, wird in „Papa, Charlie hat gesagt...“ (von Ursula Haucke, 1985) aufgegriffen und wurde für den DaF-Unterricht auch schon didaktisiert.

${ }^{8}$ http://daz-lernwerkstatt.de/glossar/halbsprachigkeit-semilingualismus/ (Zugriff: 12.06.2015)

${ }^{9}$ Denn dies ist ja unweigerlich mit dem schnellen Verlust der eigenen Sprache und Kultur verbunden, und dies sollte unter anderem durch das neue Konzept verhindert/vermieden bzw. minimiert werden.

${ }^{10}$ Obwohl ich geschrieben habe, dass die Sprache ein Element/ein Teil der Kultur ist, erwähne ich manchmal beide Begriffe, sodass wir Kultur nicht nur mit Sitten und Gebräuchen bzw. Kulturartefakten, also Kunstwerken, assoziieren, sondern auch mit allem, was mit der Sprache verbunden ist.
} 
Das Medium Film als Element mit hohem interkulturellem Potenzial 161

Sprache und ihre Kultur von Zeit zu Zeit im Mittelpunkt des Interesses stünde, und zweitens, dass die Einheimischen (in der Schule also die deutschen Mitschüler der Migrantenkinder) diese anderen Kulturen und Sprachen kennen lernen würden. Damit würde sich deren Offenheit/Toleranz vergrößern und die positive Einstellung zu dieser für sie (noch) fremden Welt verstärken.

\section{Interkulturelle Kompetenz}

Die Lehrkräfte sollten eine interkulturelle Kompetenz ${ }^{11}$ aufweisen, die zunächst einmal eine kulturelle Kompetenz (bezüglich ihrer Kultur) darstellt. Als Interkulturelle Kompetenz wird die (erlernte) Fähigkeit verstanden, „mit Individuen und Gruppen anderer Kulturen erfolgreich und angemessen $\mathrm{zu}$ interagieren, im engeren Sinne die Fähigkeit zum beidseitig zufriedenstellenden Umgang mit Menschen unterschiedlicher kultureller Orientierung. “12 Diese Fähigkeit könne schon in jungen Jahren vorhanden sein oder später im Rahmen des Enkulturationsprozesses (direkte und indirekte Erziehung) auch entwickelt und gefördert werden.

M. Byram (2001:3) stellt wichtige Aspekte der Vermittlung von Interkultureller Kompetenz folgendermaßen dar:

the emphasis on learners becoming aware of and analysing the cultural phenomena of their own society as much as those of other societies;

the development of skills of analysis and interpretation of unfamiliar social and cultural data from a foreign society; (...)

the use of literary texts to stimulate affective as well as cognitive understanding of otherness and the use of students' literary imagination.

9. Interkulturalität in der Didaktik

Die Fremdsprachenlehrkraft sollte mit der Thematik und den Problemen der Multi- und Interkulturalität vertraut sein, aber auf der

\footnotetext{
${ }^{11}$ W. Pfeiffer, 2001:148; siehe auch www.tu-berlin.de/fb2/fadi/hr/hr-ICC.htm.

$12 \mathrm{https} / / / \mathrm{de} . w i k i p e d i a . o r g / w i k i /$ Interkulturelle_Kompetenz (letzter Zugriff: 18.10.2015).
} 
anderen Seite sollte sie auch die Schüler/Studenten zum Nachdenken sowie zum Arbeiten (schriftlich, mündlich, Projekte) zu diesen Themen ermuntern können. Während des Studiums sollte die zukünftige Lehrkraft auf so eine Arbeit vorbereitet werden, sowohl emotional und sozial als auch inhaltlich. Das Thema der Interkulturalität sollte ein Teil der (universitären) Ausbildung der Fremdsprachenlehrkräfte und auch als ein allgemeines Prinzip in allen Studienrichtungen verankert sein.

Im bestmöglichen Fall könnte die Lehrkraft das Wissen/die Erfahrung der Immigrantenkinder, die mit deren Kultur/Sprache/Lebensform/Sitten/Geschichte zusammenhängen, nutzen. Wie aus dem vorhin Gesagten zu entnehmen ist, können die Lehrkräfte fast aller unterrichteten Fächer ihren Unterricht mit interkulturellen Elementen bereichern. Sogar im „trockenen“ Lateinunterricht ist es möglich, anschaulich und interessant von der alt-römischen Kultur zu berichten. Andere Kulturen/Sprachen und die Menschen aus anderen Ländern/Kulturkreisen werden somit auch geschätzt. Wenn das Wissen der Kinder nicht ausreichen sollte, kann die Lehrkraft auch die Eltern zur Zusammenarbeit einladen.

In einer Unterrichtsstunde können nicht nur eine „exotische“, also ganz fremde Sprache (für die Deutschen z. B. Polnisch) und neue Bräuche (z. B. der Weihnachtsabend des 24. Dezember in Polen) (kennen) gelernt werden, aber auch die Konflikte, die zwischen den Migranten und Einheimischen (siehe die brutalen Ausschreitungen in Frankreich Ende $2005^{13}$ ) entstehen, erörtert werden. Interkulturalität ist ja multi-dimensional, daher kann sie Inspiration für viele unterschiedliche Projekte sein. ${ }^{14}$

13 Leider gibt es etliche Beispiele neueren Datums, z. B. 2015: vgl. http://www.focus.de/politik/ausland/migration-wieder-ausschreitungen-auf-

lesbos_id_4926956.html; ein Beispiel aus Österreich: http://kurier.at/chronik/wien/wiens-balkan-meile-skandalspiel-loeste-randaleaus/91.261.355 (letzter Zugriff: 12.06.2015).

${ }^{14}$ Ein Filmprojekt dieser Art (die Aufzeichnung von eigenen Theateraufführungen) zum Thema Konflikt zwischen verschiedenen ethnischen Gruppen entwickelten Schüler einer Amsterdamer Schule. 
Das Medium Film als Element mit hohem interkulturellem Potenzial 163

10. Begegnung von Kulturen als Chance

Dem manchmal in einer Diskussion erwähnten Argument, dass eine Verständigung zwischen unterschiedlichen Kulturen (fast) nicht möglich sei, dass dies einfach nicht klappen könnte, ist entgegenzuhalten, dass dies keine angemessene Sichtweise sei, und zwar aus folgenden Gründen:

- Insbesondere in Europa existiert das Phänomen, dass Menschen, die einen unterschiedlichen kulturellen Hintergrund aufweisen, in einem Gebiet leben, es besteht einfach, die Zeit kann nicht zurückgedreht und diese Tatsache muss akzeptiert werden.

- Es sind also möglichst große Bemühungen anzustellen, um eine bestmögliche Verständigung zwischen den Menschen zu schaffen, insbesondere bei Kindern und Jugendlichen, also im Bildungssektor (Kindergarten, Schule, Hochschule); aber auch auf anderen Gebieten wären gezielte Aktivitäten vorstellbar (z. B. beim Sport ${ }^{15}$ ). Eine fruchtbare und konstruktive Kommunikation zwischen Menschen mit einem unterschiedlichen kulturellen Hintergrund wäre wünschenswert - und dies aus zwei Gründen:

- um der größten Gefahr, die uns bedrohen könnte (Konflikte u. Kriege) entgegenwirken bzw. ihnen vorbeugen zu können.

- um unsere europäische Kultur- und Sprachenvielfalt bewahren zu können; wir können diese Vielfalt manchmal vielleicht sogar vergrößern bzw. gefährdete Sprachen schützen und unsere kulturelle und Sprachenvielfalt in unserem relativ kleinen Europa genießen.

\footnotetext{
${ }^{15}$ http://www.fclangweid.de/index.php?page=8 (letzter Zugriff 18.10.2015)
} 
Um uns in Zukunft auch die Chance zu eröffnen, dass sich eine Synthese aus unterschiedlichen Kulturen (Kulturelementen) ${ }^{16}$ entwickeln könnte, und zwar so, dass die einzelnen Kulturen in der Lage sind, sich gegenseitig auch zu befruchten und etwas Neues zu schaffen ${ }^{17}$. Eine neue Idee ist oft das Ergebnis einer kulturellen Durchdringung (engl. intermingling of cultures, poln. przenikanie kultur; zum kulturellen Schmelztiegel siehe R. Utri, 2011), wenn der Mensch mit Fremdheit(en) zu tun hat ${ }^{18}$.

Hierin liegen eben auch die größten Chancen für unsere europäischen Gesellschaften - eine Verständigung zwischen den Staaten (ein stabiler Friede in Europa) wird auf Dauer auch die Basis dafür sein, dass sich Europa wirtschaftlich gut weiterentwickelt. Allerdings ist die Verständigung allein nicht die einzige Basis der Entwicklung: auch die materielle Basis (materielle Not der Ausländer, u. a. durch Ausnützung durch die Arbeitgeber, wie dies G. Wallraff bewies; zum Einkommen von Menschen mit Migrationshintergrund siehe Ch. Schurian-Bremecker 2012:25) sowie das Erlernen der demokratischen Struktur (inkl. ethische Werte, Menschenrechte, Tierschutz etc.) durch die Immigranten werden notwendig sein, diese Ziele zu erreichen. So wird der Wohlstand in den Einwanderungsländern (z. B. Deutschland, Österreich) davon abhängen, ob die Bürger dieser Länder in der Lage sein werden, mit

\footnotetext{
16 So meinte schon K. Popper (1990:134): „Vielleicht hängt diese kulturelle Produktivität mit meinem Thema zusammen, mit dem Zusammenprall von Kulturen. Das alte Österreich war ein Abbild Europas: Es barg fast zahllose sprachliche und kulturelle Minoritäten. (...) Wir wissen, dass Haydn und Mozart von deutschen, von italienischen und französischen Meistern beeinflusst waren, aber auch von ungarischer Volksmusik und sogar von türkischer Musik. Das Genie der Musiker bleibt ungeklärt, (aber) (...) es erscheint, dass (sie) in einer höchst kritischen Lage zwischen dem Osten und dem Westen, durch den Zusammenprall von Kulturen unermesslich bereichert wurden." Siehe auch: R. Utri, 2010. Inwieweit dies jedoch zu einem Kulturverlust führen würde, wäre eine eigene Diskussion wert.

${ }^{17}$ Im kulinarischen Bereich, wo heutzutage die Spitzenköche mit viel Fantasie unterschiedlichste Rezeptmischungen herstellen, wird gezeigt, dass dies nicht nur möglich ist, sondern auch von den meisten gerne angenommen wird.

${ }^{18}$ http://hum.uwb.edu.pl/ kolo/n_burszt.htm (letzter Zugriff: 17.09.2015)
} 
Das Medium Film als Element mit hohem interkulturellem Potenzial 165

ihren Nachbarn (und nicht nur) gute Beziehungen in allen Lebensbereichen (Politik, Diplomatie, Handel/Wirtschaft, Wissenschaft, Sprachen und Sprachenpolitik, Fremdenverkehr) zu unterhalten.

\section{Integration in der Diskussion}

Die Wissenschaft, insbesondere die über Sprachen, trugen schon lange zur menschlichen Integration bei; man muss jedoch auch hinzufügen, dass die Sprachwissenschaften nicht immer einen positiven Einfluss hatten: so trugen die Germanistik/ Romanistik/ Anglistik/ Polonistik auch zu einer Erweckung eines nationalen Bewusstseins bei und verstärkten so manchmal den Nationalismus ${ }^{19}$.

Das Paradebeispiel ist bekannt - die Nazis wollten nicht nur das „arische Volk“ von „unreinen“ Rassen, sondern auch deren Sprache von Fremdwörtern säubern (was allerdings auch seine Grenzen hatte; siehe auch L. Spitzer, 1918). Jetzt sei es, so F. Grucza (2003: 47), sicherlich wert festzustellen, wann die Neuphilologie positive oder negative Folgen in ihren (sprachlichen) Äußerungen hatte und ob diese traditionellen Haltungen noch zu den Bedürfnissen der heutigen Zeit passen, und auch ob sie in der Lage sind, zur Integration in Europa beizutragen. Die Integration in Europa ist ein langfristiges Projekt und jeder/jede, der/die etwas mit (Fremd-)Sprachen zu tun hat, kann durch sein Engagement dazu beitragen, dass die Integration besser funktioniert.

Diese Integration kann unterschiedliche Bereiche umfassen, z. B. die Wirtschaft, Politik/Diplomatie, die Kunst, den Fremdenverkehr, die Wissenschaft usw. Die polnische Germanistik wird es meist mit der Integration mit den Deutschen zu tun haben und muss hierin ihre wissenschaftliche Tätigkeit erweitern (vgl. F. Grucza, 2003:48ff.). Die menschliche Integration wird eine immense Bedeutung haben; diese funktioniert, wenn Menschen mit unterschiedlichem kulturellen

\footnotetext{
${ }^{19}$ Vgl. F. Grucza (2003:46ff)
} 
Hintergrund sich gegenseitig kennen lernen, verstehen lernen und gleichzeitig Vorurteile und Stereotypen ablegen (vgl. F. Grucza, 1994).

Solche Themen sollten auf allen Ebenen besprochen werden, in verschiedenen Institutionen (Bildungseinrichtungen). Toleranz bezüglich unterschiedlicher Lebensbereiche und die Akzeptanz von anderen Standpunkten und Sitten - falls diese nicht in die Freiheitsbereiche von anderen Menschen eindringen - wird nötig sein. Eine vollständige Assimilation, also der Verzicht auf die eigene Tradition/ Muttersprache tritt selten in Erscheinung, eher nach zwei bis drei Generationen (ein Immigrantenkind, das schon in Deutschland geboren ist, kennt kaum mehr die Sprache und das Herkunftsland seiner Großeltern; die Statistik zeigt, dass $50,3 \%$ der Personen mit türkischem, 45,8\% mit italienischem und 41,0\% mit griechischem Migrationshintergrund nicht selbst nach Deutschland zugewandert sind) ${ }^{20}$.

Die Integration verläuft stufenweise. Der Unterschied zwischen dem Ausmaß, in dem der Immigrant neue Gepflogenheiten (Sprache) annimmt, und andererseits zwischen dem Ausmaß, in dem die Einheimischen wollen, dass der Immigrant die neuen Sitten ihres Landes annimmt und auf seine alten kulturellen Elemente verzichtet, bestimmt die Wahrscheinlichkeit des Auftretens von Kulturkonflikten. Oft sehen die Einheimischen die Fremden nur als billige Arbeitskräfte (und die Immigranten spüren bzw. wissen das) ${ }^{21}$, leben nur in ihrer

20 Vgl. weitere statistische Angaben im Migrationsbericht: http://www.bamf.de/SharedDocs/Anlagen/DE/Publikationen/Migrationsberichte/migr ationsbericht-2012.pdf? blob=publicationFile; Näheres zu Migration und Integration schreibt R. Geißler, online unter: http://www.bpb.de/izpb/198020/migration-undintegration? $\mathrm{p}=$ all (letzter Zugriff: 24.062015).

${ }^{21}$ G. Wallraff, ein deutscher Journalist, hat das Experiment gewagt sich als Türke ausgegeben und zwei Jahre lang unter Gastarbeitern (übrigens nie angemeldet!) gearbeitet. Seine Erfahrungen hat er in seinem Werk „Ganz unten“ veröffentlicht (dies ist auch auf Youtube dokumentiert). Zu seinem Experiment siehe auch R. Utri, 2010. Weitere Berichte über das Ausnutzen von Immigranten siehe online unter: http://www.rp-online.de/nrw/duisburg- 
Tradition und interessieren sich überhaupt nicht dafür, welche Einstellungen die Immigranten vertreten, welche Erfahrungen/ welches Wissen sie besitzen oder auch wie sie leben.

S. P. Huntington (2008, Clash of Civilisations) spricht davon, dass zwei Kulturen zusammenprallen - in Wirklichkeit treffen sich Menschen mit ihrer kulturellen Erfahrung, mit ihrer Idiokultur/ihrem Idiolekt ${ }^{22}$, erfahren einen Kulturschock oder es kommt aufgrund unterschiedlicher Gebräuche, Einstellungen oder infolge von sprachlicher Unkenntnis zu Missverständnissen.

12. Film als Medium mit (inter)kulturellem Potenzial

Ein Medium, mit dem man auf Kulturunterschiede hinweisen und mit dem man die sich daraus ergebenden Konflikte aufzeigen kann, ist der Film. Filme zeigen oft auf humorvolle Weise, wie sich zwei Kulturen treffen und welche Probleme/Missverständnisse dadurch entstehen (können). Der Zuseher, der sich in die Rolle des Helden/Immigranten - hineinversetzt, sieht nun die Welt aus anderer Sicht. Dies könnte zur Reflexion über Diskriminierung, Kulturschock sowie zum Dialog zwischen den Kulturen anregen. Das Medium Film erweckt eben oft auch Empathie und bringt uns den Helden näher, was dazu führt, dass es leichter ist, die Elemente der anderen Kultur zu verstehen und gegebenenfalls zu akzeptieren (vgl. M. Byram, G. Zarate, 1995: 11).

13. Immigrantenfilme

Es gibt schon etliche Filme, die auch von Immigranten(kindern) selbst gedreht wurden, z. B. von Italienern in den USA, von Algeriern in Frankreich oder von Türken in Deutschland. Auch Einheimische sehen es manchmal als Herausforderung, einen Film zu schaffen, der interkulturelle Konflikte und Lösungen zum Hauptthema des Filmes

auslaender-brutal-ausgenutzt-aid-1.1327144;

https://www.neuesdeutschland.de/artikel/525528.auslaender-durch-firmen-ausgenutzt.html; http://www.ndr.de/nachrichten/niedersachsen/Ausgenutzt-Lohnsklaven-inNiedersachsen,billigloehner101.html (letzter Zugriff: 15.10.2015).

${ }^{22}$ Vgl. F. Grucza (1992: 14, 16 u. 47 ff.), sowie R. Utri (2007b: 75). 
macht. So zeigt der österreichische Regisseur Wolfgang Murnberger im Film "Kebab mit alles" den biederen Wiener Kaffeehausbesitzer Johann Stanzerl (übrigens wunderbar gespielt von Andreas Vitasek), der mit Türken in formellen und personellen Konflikt kommt ${ }^{23}$. Auch der deutsche Regisseur Rainer Werner Fassbinder hatte schon in den 1970er- Jahren den Mut gezeigt, im Film „Angst essen Seele auf“ einen Marokkaner als Hauptfigur eines Filmes zu zeigen ${ }^{24}$.

Türkische Regisseure zeigten das Land Deutschland als ein unsympathisches und den Gastarbeitern nicht geneigtes. „In den 1970er und 1980er Jahren waren die (wenigen) deutsch-türkischen Filme noch geprägt von ernsten Geschichten über Migration und die oft damit einhergehenden Krisen und Schwierigkeiten“" ${ }^{\text {25. Die }}$ „türkischen“ Regisseure (Immigrantenkinder) präsentieren das Leben der Türken aus der Sicht der „Misafir işçi“ (Gastarbeiter) ${ }^{26}$.

14. Weiterentwicklung der Immigrantenfilme

Filme wie "Gegen die Wand" (2004), "Soul kitchen" (2009) oder „Auf der anderen Seite" (2007) (alle von Fatih Akin) haben schon eine größere Distanz zur Immigrationsgesellschaft, sie erzählen ihre Geschichte bis zum Ende. Die pluralistische deutsche Gesellschaft ist offensichtlich schon so reif, dass man den Zusehern vor der Leinwand bzw. vor dem Bildschirm solche Filme zumuten kann.

Von dem oben Gesagten schlussfolgere ich, dass das interkulturelle Lernen (die Erhöhung der interkulturellen Kompetenz) zum Hauptthema werden kann, wenn man mit dem Medium Film im DaF-

\footnotetext{
${ }^{23}$ Der Film wurde auch prämiert: Er bekam den Fernsehpreis für Erwachsenenbildung 2012 und beim 18. Shanghai TV-Festival 2012 den Magnolia Award für "Bestes Drehbuch" und "Beste Regie".

${ }^{24} \mathrm{Vgl}$. http://www.fassbinderfoundation.de/movies/angst-essen-seele-auf/

${ }^{25} \mathrm{http}$ ://blog.maxdome.de/kino-film/kino-zwischen-zwei-kulturen-tuerkischdeutsche-filmemacher (letzter Zugriff: 28.06.2015)

${ }^{26}$ So z.B. auch Tevfik Basers "40 qm Deutschland" (aus dem Jahre 1985; dieser Film zeigt türkische Frauenschicksale und prangert Menschenrechtsverletzungen an) oder Hark Bohms "Yasemin" (1988; dieser Film setzt sich mit den kulturellen und religiösen Gegensätzen zwischen Einheimischen und Zuwanderern auseinander).
} 
Das Medium Film als Element mit hohem interkulturellem Potenzial 169

Unterricht arbeitet. Diesmal möchte ich die Problematik anhand zweier Filme darstellen: Solino, auch von Fatih Akin 2002 produziert $^{27}$ ), und I love Vienna, ein österreichischer Film von Houchang Allahyari aus dem Jahre 1991.

15. Der Film Solino (aus dem Jahre 2002):

In diesem Film werden 20 Jahre einer süditalienischen Familie erzählt, die 1964 ins Ruhrgebiet emigrierte und die erste Pizzeria in Duisburg als Familienbetrieb eröffnete. Fatih Akin erzählt hier eine Geschichte, er erzeugt Stimmungen, die das Leben der italienischen Einwanderer einfangen (so z.B. die Szene, wo die Mutter einen Vogel im Zimmer entdeckt: „Ein Vogel im Haus bringt Unglück, wir müssen ihn einfangen!“ Oder die Frage des Kindes: „Wieso schneit es nicht? Wir sind doch in Deutschland!").

Da dürfen weder romantische (z.B. die Diskussion darüber, wie sie ihr neues Restaurant benennen sollen: „Solino - wie bei uns zu Hause!" Darauf gibt er ihr einen Kuss) noch tragikomische Episoden fehlen (z.B. das Gespräch des Vaters mit seinen Söhnen, die nicht in seine Fußstapfen treten wollen: „Es gibt mehr als Pizza auf der Welt.“ „Was denn, zum Beispiel?“ „Feuer, und Leidenschaft.“ „Und was kannst du dir davon kaufen, von Feuer und Leidenschaft?" Darauf stehen beide Söhne wortlos auf und gehen.

Natürlich sind die Charaktere (vor allem der beiden Brüder, wo einer ein sensibler Künstler ist, der andere einen Verbrecher darstellt) übertrieben dargestellt, es werden also Klischees vorgegeben, die jedoch durch die Überzeichnung nicht mehr realistisch aussehen.

16. Didaktisierung des Filmes:

Dieser Film hat eine interkulturelle Bedeutung, und zwar aus drei Gründen:

${ }^{27}$ Dieser Film wurde auch schon im Rahmen einer Seminararbeit didaktisiert; vgl. Jenny Tippelt, 2011. 
i) Er zeigt die Unterschiede zwischen den Ländern/Kulturen (Deutschland/Italien), verschiedene Sitten und Gebräuche, unterschiedliches Verhalten von Menschen in diversen Situationen.

ii) Am Beispiel der italienischen Familie ist der Konflikt einerseits durch den Generationsunterschied (Söhne Vater), als auch dadurch motiviert, dass die Söhne im liberalen Deutschland aufwachsen und Elemente dieser neuen Kultur übernehmen, zum Teil jedoch auf ihre spontane italienische Art und Weise.

iii) Immer wieder gibt es Szenen aus dem armen und heruntergekommenen Süditalien, das jedoch auf den künstlerischen Sohn wirkt, indem er dorthin fährt (auch wenn er vom hinterhältigen Bruder dorthin gelockt wird) und dafür auch eine gewisse Faszination entwickelt. Dieses ,innere Zerrissen-Sein“ zwischen dem Heimatland der Eltern und dem modernen Deutschland ist ein interkulturelles Thema schlechthin und wahrscheinlich auch das wichtigste Element für den Szenenwechsel in diesem Film.

Wenn sich eine Lehrkraft dafür entscheidet, diesen Film den Schülern/Studenten $^{28}$ zu zeigen, sollte sie sich vorher überlegen, welche Fragen sie ihren Deutschlernern nach dem Film stellen wird. Sie sollte sich mit der Problematik schon ein wenig beschäftigen wenn sie interkulturelle Veranstaltungen verfolgt, wird sie kompetent genug sein, mit ihren Schülern/Studenten darüber zu diskutieren ${ }^{29}$.

Ein lohnenswerter Ansatz zur Arbeit mit einem Immigrantenfilm zeigt U. Reeg (vgl. 2008: 123), die Lernaktivitäten für literarische Texte vorstellt; diese können auch auf den Film umgelegt werden: 1. Phase: den Lernenden wird ein Denkanstoß gegeben

\footnotetext{
${ }^{28}$ Falls ich nicht immer die weibliche Form verwende, soll dies nicht aus dem Grund der Negierung der feministischen Errungenschaften in der Sprache sein, sondern damit nicht zu viel textliche Unruhe gestiftet wird und der Text noch leicht lesbar bleibt.

${ }^{29}$ Siehe R. Utri (2007: 177 ff.)
} 
Das Medium Film als Element mit hohem interkulturellem Potenzial 171

2. Phase: die Lernenden beobachten den Film mittels Leitfragen (und problematisieren damit die interkulturelle Kontaktsituation)

3. die Lernenden entwerfen Veränderungen im Handlungsverlauf

4. Die Lernenden spielen Rollenspiele und spielen die Handlung nach bzw. schreiben eigene Dialoge, sie erfinden Personen, die für die Interkulturalität eine Funktion ausüben.

Das interkulturelle Lernen sollte eigentlich einen Teil des Studiums darstellen, insbesondere wenn das Studium mit Sprachen oder Kulturen zu tun hat. Das Medium Film ist ein didaktisches Material, das den Vorzug hat, dass die Schüler/Studenten sich entspannter als in anderen Unterrichtsstunden fühlen. Das Lernen ist somit eine Art Mußezeit (siehe dazu K. Geißler sowie R. Utri, 2007b:134ff.) Wenn die Sprachenlerner/innen sich einen Film ansehen, dann kann die Lehrkraft sie sprachlich besser aktivieren als in Unterrichtsstunden, die auf rein sprachlichen Übungen basieren.

17. Der Film I love Vienna (aus dem Jahre 1991)

Der im Iran geborene Regisseur Houchang Allahyari 1991 hat ein großartiges Schauspielerteam um sich versammelt (Fery Farokhzad, Dolores Schmidinger, Hanno Pöschl, Marisa Mell und andere) und zeigt eine Problematik die auch bis heute in Wien (Österreich, aber auch in den meisten mittel- und westeuropäischen Ländern) aktuell ist. Auch in anderen Filmen (z. B. „Geboren in Absurdistan“, 1999) thematisiert er die Schicksale von Flüchtlingen, Asylsuchenden und Migranten. Dies macht er auf dramatische Weise, allerdings sind seine Filme auch mit viel Humor gespickt.

Die Problematik läuft zweischienig: einerseits die Probleme, mit denen sich Immigranten, in diesem Fall Ali Mohammed und sein Sohn sowie seine Schwester, herumschlagen müssen, als sie in Wien, das schon seit langem eine multikulturelle Metropole ist, landen. Bis heute ist es jedoch nicht unbedingt ausländerfreundlich ${ }^{30}$. Auf der

\footnotetext{
${ }^{30}$ Dies wurde im Jahre 2013 sehr deutlich, als Asylbewerber die Votivkirche in Wien besetzten und Dinge (und die Caritas unterstützte dies) forderten, die der Staat den Flüchtlingen jahrelang vorenthalten hatte: „Grundversorgung für alle Asylwerber,
} 
anderen Seite kommen Österreicher zum Zug, die mit ihnen zu tun haben und ihre Lebenseinstellungen zeigen. Im Film werden verschiedene Einstellungen zum Helden gezeigt, und zwar:

i) Freundlichkeit, Entgegenkommen und Wohlwollen den Ausländern gegenüber - am Anfang tritt ein Bekannter in Erscheinung, der für Ali einen Aufenthalt in einem billigen Hotel organisiert hat (Hotel Praterstern) sowie eine Aufenthaltsgenehmigung und später auch ein Visum in die USA; die Gastgeberin, bei der Ali später privat wohnt, die sich sehr um ihn sorgt; auch die Ex-Frau des Hotelbesitzers, die sich in Ali Mohammed verliebt und daher seine mohammedanischen Sitten (,Das ist ein Huhn - islamisch geschlachtet."), seine Religion und seine spezifische Cuisine, seinen Akzent und seine kleinen sprachlichen Fehler (Ali war im Iran Deutschlehrer) respektiert und im Umgang mit ihm berücksichtigt. Damit zeigt sie ihm ihr großes Herz (,das goldene Wienerherz”). Der Pole Karol Tarnowski, der Kommunismusflüchtling ist und im selben Hotel wohnt, verliebt sich in Alis Schwester und damit er sie heiraten kann, heiratet er sogar auf islamische Art.

ii) Gleichgültigkeit, Indifferenz und Kälte sowie auch eine scheinbare Freundlichkeit - der Hotelbesitzer nimmt Immigranten (Asylwerber) als Hotelgäste auf und damit auch die mit ihrer Fremdheit verbundenen Schwierigkeiten. Meistens versucht er sie ein wenig zu beschwindeln (er befiehlt dem Kellner die Verteilung von kleineren Frühstücksportionen). Wenn sie jedoch zu viele Probleme machen (so sieht Karol die Ausnutzung, schreibt einen Beschwerdebrief und sammelt

unabhängig vom Rechtsstatus, freie Wahl des Aufenthaltsortes, Zugang zum Arbeitsund Wohnungsmarkt sowie die Einrichtung einer Instanz zur Überprüfung aller negativ beschiedenen Asylverfahren.“ Vgl. http://kurier.at/chronik/votivkirchefluechtlinge-stellen-forderungen/1.977.614 (letzter Aufruf: 28.06.2015) 
Unterschriften), ist er bereit, sie hinauszuwerfen (,Du kannst ja wieder nach Hause gehen, der Osten ist ja jetzt wieder offen!"), da er sicher ist, bald wieder neue Gäste für sein Hotel, das sich auch nicht in der besten Lage befindet (in unmittelbarer Nachbarschaft befindet sich eine lärmmachende Autowerkstatt sowie ein Bordell), zu gewinnen. Auch tritt ein Rechtsanwalt in Erscheinung, zu dem Ali Mohammed sich mit seinem österreichischen Freund zwecks Aufenthaltsgenehmigung begibt. Dieser elegante Dr. Borsodi - selbst auch ein Immigrantenkind (!) - sollte als Rechtsanwalt nichts Illegales tun. Wenn er jedoch finanziellen Gewinn machen kann, ist er $\mathrm{zu}$ Unehrlichkeiten bereit: so empfiehlt er Ali, sich als Christen auszugeben, der in seinem Heimatland verfolgt würde, damit sein Asylverfahren positiv ausfalle. Er spricht sachlich, auf Englisch und zeigt sich nicht einfühlsam. Als Ali erklärt, warum er in Wien bleiben wolle und auch I love Vienna hinzufügt, sagt er nur trocken: „Das ist aber kein Grund, eine Genehmigung zu bekommen!"

iii) Arroganz, Hochnäsigkeit, Eingebildetheit den Ausländern gegenüber; diese Einstellung ist schon ,ausländerfeindlich”; verkörpert von den BordellProstituierten, welche die Immigranten von oben herab behandeln, höchstens als potentiell naive Kunden betrachten und über sie lachen; auch der Hotelbesitzer, der sich nur um Ordnung sorgt und keine nicht-westlichen Verhaltensweisen erlaubt (,Sie können doch nicht im Pyjama da an der Rezeption stehen und so einen Lärm machen!"); er versucht gar nicht, Hochdeutsch zu sprechen, was den Ausländern natürlich Schwierigkeiten bereitet; er spricht mit Ali sehr ironisch („Wenn Sie mit meiner Frau geschlafen haben, sind Sie bestraft genug!") und mag Karol nicht mehr, als er über das miese Frühstück schimpft. In dieselbe Kerbe schlägt auch die 
Wiener Polizei, die, als sie jemand wegen Ruhestörung ruft, schnell den Schuldigen findet - den Sohn von Ali, der nach dem Böllerschießen allein auf der Straße geblieben war. Die Polizei schnappt ihn und hat damit die Bestätigung, dass immer die Ausländer die Unruhestifter sind (sein Vater muss ihn aus der U-Haft holen).

Im Film werden auf humorvolle Weise die Problemchen gezeigt, die mit dem Ausländersein in Wien verbunden sind. Der Zuschauer sieht Wien als buntes Nest, wo sogar ein Japaner und ein Schwarzer im Spital arbeiten. Trotzdem fühlt sich Ali anfänglich sehr fremd. Er behält seine Sitten bei - er betet (als jemand morgens läutet: „Entschuldige, ich habe gebetet.” - „Hätten Sie nicht später weiterbeten können?” - „Nein, das dürfen wir nicht!”), er trinkt keinen Alkohol (sein erster Konsum wird Ende des Filmes auch genutzt), hat seine Moral (zur Frau des Hotelbesitzers nach einer intimen Nacht: „Was du mit mir gemacht hast, das macht eine gute Frau nicht!") und bleibt seiner Kultur treu (orientalische Musik). Auch bei der Religion bleibt er stur: Karol muss sich in sein Schicksal fügen und den Islam annehmen, Ali schlägt ihm einen neuen Vornamen vor: Abdullah Karol Tarnowski. Er hat die unter Moslem übliche Beziehung zu den Frauen - er passt auf seine Schwester auf, wie wenn sie seine Tochter wäre und als diese eine eigene Meinung äußert, erleidet er einen leichten Schock (später noch einmal, als sie beim Tanzen das Tuch wegmacht und ihr Dekolleté zeigt: „Wie siehst du denn aus?"). Sogar gegen Ende des Films, als er sich dazu entscheidet, bei Frau Swoboda zu bleiben, hat er noch Zweifel: „Was soll ich hier machen in Wien? Ich kann doch nicht von dir leben". Er hat kaum Aussicht auf eine Arbeit und muss von ihr leben, was jedoch gegen seine Überzeugung ist.

Aber nicht nur Kultur-Komparatisten und Unterhaltungssuchende, sondern auch Linguisten kommen bei diesem Film auf ihre Kosten. Ali bessert manchmal sogar die Österreicher aus, die zu ihm wie zu einem primitiven Gastarbeiter sprechen (,Du sprechen gut Deutsch”er antwortet: „Sie aber nicht!”) oder die zu ihm in wienerischer Umgangssprache sprechen: „Du kannst mir ja etwas lernen!” 
(Prostituierte von gegenüber) - er: „Das heißt ,mich lehren', nicht mir lernen."

Andererseits tritt Ali auch des Öfteren ins Fettnäpfchen: statt „im wehrpflichtigen Alter" sagt er ,im wehrfähigen Alter”; als er Schimmelkäse kauft, meint er: „Die haben mir schlechten Käse verkauft!“ und Frau Swoboda muss ihm den Unterschied zwischen „Schimmelkäse“ und „,verschimmelter Käse“ erklären. Als er vom Arzt heimkommt, erzählt er: „Ich habe Hypochonder, aber das ist gar nicht so schlimm!"

Der Film zeigt auf heitere Art und Weise, dass jeder etwas von einer anderen Kultur lernen kann. Am Ende feiern die Österreicher mit den Immigranten bei orientalischer Musik, der Iraner Ali akzeptiert die Hochzeit seiner Schwester mit dem Polen Karol, seinem Sohn gefällt das lockere Leben in Wien und lernt schnell die Sprache. Schließlich resümiert Ali mit einer gewissen Melancholie: „Irgendwie hat die Zeit in Wien alles verändert, Wien hat mich übergerollt (noch ein kleiner sprachlicher Ausrutscher), soviel ist zerbrochen." Aber der Tanz, zu dem er sich aufrafft, befreit ihn von allem Frust. Auch der Kollege sieht alles positiv, sogar als man ihm den Blinddarm entfernt: „Ich hab mir gedacht, er kann sich von Wien nicht trennen, ein kleines Stückerl bleibt da.“

Zusammenfassend lässt sich feststellen: Alle Filme, die dazu anregen, Rassismus, Diskriminierung, Kulturunterschiede und Konflikte zu reflektieren, können beim interkulturellen Lernen im DaF-Unterricht hilfreich sein. Wenn die Lehrkraft sich entsprechend vorbereitet und sich die Mühe macht und den Film didaktisch entsprechend aufbereitet, ist es höchstwahrscheinlich, dass sie ihre Schüler bzw. Studenten auf interkulturelle Begegnungen optimal vorbereiten kann.

Literatur:

Brown, M., 1997, Turning Points. Essays in the History of Cultural Expressions. Stanford University Press.

Byram, M., Zarate, G. 1995, Young People Fading Difference: Some Proposals for Teachers. Council of Europe 1995 
Byram, M./Nichols, Adam/Stevens, David (Hrsg.), 2001, Developing Intercultural Competence in Practice. Clevedon etc.: Multilingual Matters Ltd

Creemers, B. P. M. 1999, Educational effectiveness and improvement in developing societies. Some experiences from the primary Education Quality Improvement in Indonesia. In: Tertium comparationis 5, 32-51.

Geißler, K.A.: „Ich hinke etwas der Zeit voraus.“ Interview mit Zeitforscher Karlheinz A. Geißler über die Kosten des Tempowahns und die Notwendigkeit, Zeit als Wohlstandsindikator zu begreifen. http://www.stern.de/reise/reise-ichhinke-etwas-der-zeit-voraus-509202.html, Zugriff 13.06. 2015.

Güngör, D., 2007, Ganz schön deutsch. Meine türkische Familie und ich. München: Piper-Verlag.

Grucza, F. 1992, Kulturowe determinanty języka oraz komunikacji językowej. In: F. Grucza (Hrsg.), Język, Język, kultura - kompetencja kulturowa. Warszawa, 9-70

Grucza, F. (Hrsg.) 1992, Język, kultura - kompetencja kulturowa. Materiaty z XIII Sympozjum zorganizowanego przez Instytut Lingwistyki Stosowanej UW (Zaborów, 5/8 listopada 1987r.), Warszawa

Grucza, F. (Hrsg.) 1994, Uprzedzenia między Polakami i Niemcami. Materiaty polsko-niemieckiego Sympozjum Naukowego (Görlitz/Zgorzelec, 9/11 grudnia 1992), Warszawa

Grucza, F. (Hrsg.) 2003, Deutsch-polnische und gesamteuropäische Integration in Forschung, Lehre und Öffentlichkeitsarbeit der (polnischen) Germanistik. Materialien der Jahrestagung des Verbandes Polnischer Germanisten 02.-04. Mai 2003

Haucke, U. 1975, Papa, Charly hat gesagt... Reinbek bei Hamburg: Rowohlt TBVerlag.

Huntington, S. P. 2008: Zderzenie cywilizacji (Orig. The Clash of Civilisations), Warszawa: Warszawskie Wydawnictwo Literackie.

Lemmen, Th., Miehl, M. 2001: Islamisches Alltagsleben in Deutschland. Bonn: Friedrich-Ebert-Stiftung

Mackiewicz, M. (Hrsg.) 2005, Dydaktyka języków obcych a kompetencja kulturowa i komunikacja interkulturowa. Poznań: Wydawnictwo Wyższej Szkoły Bankowej

Mucha, A., 2014: Ausgewählte Aspekte interkulturell bedingter Missverständnisse Implikationen für das Fremdsprachenlehren und -lernen. In: Lublin Studies in Modern Languages and Literature 38 (1), 155-165.

Nowak, J., 2012, Interkulturalität statt Leitkultur und Assimilation Missbrauch von Begriffen statt Anerkennung sozialer Realitäten. Die Gaste (Hrsg.) 2012: Alman Eğitim Sistemi, Entegrasyon Politikaları ve Göçmenler Sempozyumu (Deutsches Bildungsystem, Integrationspolitiken und Migration - Symposium). Online unter: http://j-nowak.de/fileadmin/pdf/interkulturalitaet nowak 2012.pdf.

Pfeiffer, W. 2001, Nauka języków obcych. Od praktyki do praktyki. Poznań: Wagros 
Das Medium Film als Element mit hohem interkulturellem Potenzial 177

Pomorski, J. M. 2006, Jak narody porozumiewaja się ze sobq $w$ komunikacji międzykulturowej $i$ komunikowaniu medialnym, Kraków: Universitas

Quane, A./ Singh, M. 2004, Large scale assessments and their impact for education in the South. In: ZEP: Zeitschrift für internationale Bildungsforschung und Entwicklungspädagogik 27, Nürnberg: Waxmann-Verlag, 2-8.

Popper, K. R. 1990, Über den Zusammenprall von Kulturen; Vortrag anlässlich des 25. Jahrestages des österreichischen Staatsvertrages; hier aus: ders.: Auf der Suche nach einer besseren Welt. Vorträge und Aufsätze aus dreißig Jahren. München: Piper Verlag, 127-136.

Reeg, Ulrike, 2008: Interkulturelle Kommunikation und Fremdwahrnehmung: Literarische Texte im Unterricht Deutsch als Fremdsprache. In: Ulrike A. Kaunzner (Hg.): Der Fall der Kulturmauer. Wie kann Sprachunterricht interkulturell sein? Münster et al.: Waxmann Verlag, 117-133.

Schurian-Bremecker. Ch. (Hrsg.), 2012, „Eigentlich mehr so Deutschland, aber Türkei ist auf jeden Fall auch dabei..." Heimaterleben aus der Sicht von Jugendlichen in Deutschland. Migration und Gegenwart. Kassel: University Press.

Spitzer, L. 1918, Fremdwörterhatz und Fremdvölkerhaß. Eine Streitschrift gegen die Sprachreinigung. Wien: Manzsche Hof-, Verlags- und Universitäts-Buchhandlung

Tippelt, J. 2011, Fatih Akins Film »Solino« im Fremdsprachenunterricht, München: GRIN Verlag

Utri, R. 2007a, Die multilinguale und multikulturelle Gesellschaft. In: Grucza, S., Kornacka, M., Marchwiński, A. (Hrsg): W kręgu teorii i praktyki lingwistycznej. Księga jubileuszowa poświęcona Profesorowi Jerzemu Lukszynowi z okazji 70. rocznicy urodzin; Warszawa: Katedra Języków Specjalistycznych, 337 - 358

Utri, R. 2007b, Interkulturowość a uczenie języków obcych w Austrii, Niemczech $i$ Polsce na tle polityki językowej Unii Europejskiej. Nieopublikowana rozprawa doktorska, Katedra Języków Specjalistycznych,Warszawa

Utri， R. 2010a，Deutsch-türkische Freundschaft: über die Notwendigkeit des Einbindens der Migrantenliteratur in das Interkulturelle Lernen. In: Glottodidactica, 36, 211-223.

Utri, R. 2010b, Interkulturalität - Reflexion der Kultur und Mentalität Schlussfolgerungen für die Glottodidaktik, in: Kazimiera Myczko (Hrsg.): Reflexion als Schlüsselphänomen der gegenwärtigen Fremdsprachendidaktik,Posener Beiträge zur Germanistik (hrg. v. Cz. Karolak), Band 27, Frankfurt am Main: Peter Lang Verlag, 153-168

Utri, R. 2010c, Migrantenliteratur im DaF-Unterricht: Potenzial für das interkulturelle Lernen, in: Lingwistyka Stosowana / Applied Linguistics / Angewandte Linguistik, 3, 279-290. 
Utri, R. 2011, Flohmärkte als Schmelztiegel der Kulturen - Gedanken nach einem interkulturellen Spaziergang mit einem Seitenblick auf die Sprachenpolitik einer multikulturellen Gesellschaft und die Möglichkeiten einer zeitgemäßen Schule, in: Lublin Studies in Modern Languages and Literature, 35, 130-143.

Utri, R. 2013, Das interkulturelle Potenzial von Karikaturen im DaF-Unterricht. Glottodidactica, 40(2), 35-45.

Wallenborn, M., 2007, Interventionen in (Bildungs-)Systeme durch die Entwicklungszusammenarbeit. Ein Beitrag zur Wirkungsdebatte. In: ZEP: Zeitschrift für internationale Bildungsforschung und Entwicklungspädagogik 30, 23-27

Wallraff, G., 1985, Ganz unten. Köln: Kiepenheuer \& Witsch

Internetresources:

http://www.immi.se/intercultural/

http://www.uni-koeln.de/ew-fak/paedagogik/interkulturelle/publikationen/bern.html http://www.film.at/i_love_vienna/

http://www.isoplan.de/aid/index.htm?http://www.isoplan.de/aid/2004-1/medien.htm http://www.tu-berlin.de/fb2/fadi/hr/hr-ICC.htm

http://www.nkjociechanow.edu.pl/images/papa_hat_nichts_gegen_italiener_3.pdf http://kurier.at/chronik/wien/verzweifelte-asylwerber-im-servitenkloster-es-war-einfehler-die-votivkirche-zu-verlassen/7.444.152

http://www.welt.de/debatte/kommentare/article144801381/Warum-wir-ueber-die-

Leitkultur-reden-muessen.html 